\title{
-94 ATTG Insertion/Deletion Polymorphism of the NFKB1 Gene Is Associated with Coronary Artery Disease in Han and Uygur Women in China
}

\author{
Yi-Ning Yang, ${ }^{1-3, *}$ Jin-Yu Zhang, ${ }^{1,2, *}$ Yi-Tong Ma, ${ }^{1,2}$ Xiang Xie, ${ }^{1,2}$ Xiao-Mei Li, ${ }^{1,2}$ Fen Liu, ${ }^{1,2}$ \\ Bang-Dang Chen, ${ }^{1,2}$ Xing-Hui Dong, ${ }^{1,2}$ Ying-Ying Zheng, ${ }^{1,2}$ Shuo Pan, ${ }^{1,2}$ \\ Hui Zhai, ${ }^{1,2}$ Dong-Ze Li, ${ }^{1,2}$ and Qing-Jie Chen ${ }^{1,2}$
}

Objectives: The nuclear factor kappa-light-chain enhancer of activated B cells (NF- $\kappa \mathrm{B})$ signaling pathway plays a key role in the regulatory network of inflammation. The deletion variant allele of the NFKB1-94 insertion/deletion (ins/del) ATTG promoter polymorphism results in lower transcription levels of the p50 subunit, and the variant allele has been associated with several inflammatory diseases as well as with coronary artery disease (CAD) with inflammation playing an important part in the pathogenesis. The aim of the present study was to assess the association between the human NFKB1 gene polymorphism and CAD in a Han and Uygur population of China. Methods: We used the following two independent case-control studies: a Han population (633 CAD patients and 616 control subjects) and a Uygur population (437 CAD patients and 356 control subjects). All participants were genotyped for the same one single nucleotide polymorphism (SNP) (rs28362491) of the NFKB1 gene, that is, DD, ATTG deleted homozygote; ID, ATTG inserted and deleted heterozygote and II, ATTG inserted homozygote by real-time polymerase chain reaction. Results: The distribution of the SNP (rs28362491) genotypes was significantly different between CAD and control participants in women of the Han $(p=0.029)$ and the Uygur $(p=0.032)$ populations, but not in men. Further, DD carriers of the SNP in the NFKB1 gene were more frequent in female CAD patients than in controls in both the Han $(23.2 \%$ vs. $13.5 \%, p=0.009)$ and the Uygur $(19.8 \%$ vs. $8.3 \%$, $p=0.012$ ) population. The significant difference between DD and ID + II genotypes was retained after adjustment for covariates (for Han, odds ratio [OR]: 1.805, $p=0.029$ and for Uygur, OR: 3.192, $p=0.011$ ). Conclusions: The DD genotype of the SNP (rs28362491) in the NFKB1 gene may be considered a genetic marker of CAD in Han and Uygur women in China.

\section{Introduction}

C ORONARY ARTERY DISEASE (CAD) is an important cause of morbidity and mortality in countries all over the world. Inflammation exacerbates a number of harmful effects on the heart and, in particular, CAD. Inflammation plays a crucial role in characterizing the formation of atheromatous plaque, as well as its progression. The secretion of pro-inflammatory cytokines from the vascular endothelium and macrophages induces the production of inflammatory molecules that are measured in the circulation, such as C-reactive protein, serum amyloid A, interleukin-1beta (IL-1 $\beta$ ), tumor necrosis factor alpha (TNF- $\alpha)$, interferon gamma, IL-6, and fibrinogen (Libby et al., 2002; Kampoli et al., 2009; Vogiatzi et al., 2009).
Nuclear factor kappa-light-chain enhancer of activated B cells $(\mathrm{NF}-\kappa \mathrm{B})$ is the general name for a family of transcription factors consisting of five members: RelA (p65), c-Rel,

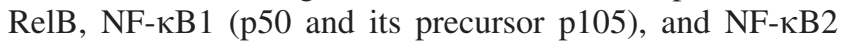
(p52 and its precursor p100). Although many dimeric forms of $\mathrm{NF}-\kappa \mathrm{B}$ have been detected, the main form of NF- $\kappa \mathrm{B}$ is a heterodimer of the p50 and p65 subunits (Chen et al., 1999). The number of genes shown to be regulated by NF- $\kappa \mathrm{B}$ is more than 160 , whereas the number of factors shown to induce the $\mathrm{NF}-\kappa \mathrm{B}$ activation is even higher (Shapshak, 2012). Numerous association studies have reported that $\mathrm{NF}-\kappa \mathrm{B}$ is a major transcription factor which regulates various cellular biological processes, including inflammatory responses, apoptosis, and cell survival and proliferation (De Winther et al., 2005; Hajer et al., 2008). Activation of NF- $\mathrm{BB}$ has been shown in many

\footnotetext{
${ }^{1}$ Department of Cardiology, First Affiliated Hospital of Xinjiang Medical University, Urumqi, China.

${ }^{2}$ Xinjiang Key Laboratory of Cardiovascular Disease Research, Urumqi, China.

${ }^{3}$ The Institute of Clinical Research, Xinjiang Medical University, Urumqi, China.

*These authors contributed equally to this work.
} 
cardiovascular diseases, including myocardial ischemia/ reperfusion injury, myocardial infarction, vein graft disease, cardiac hypertrophy, hypertension, dilated cardiomyopathy, and heart failure (Zhou et al., 2009; Dhingra et al., 2010; Santos et al., 2010; Van der Heiden et al., 2010). Atherosclerosis is recognized as a chronic inflammatory disease of the

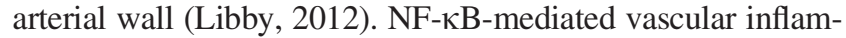
mation plays a critical role in the initiation and progression of atherosclerosis. Therefore, activation of NF- $\kappa \mathrm{B}$ may be considered an important contributor to the development of CAD.

NFKB1 (encoding for NF- $\mathrm{kB}$ ) maps to chromosome 4q23q24, consists of 24 exons, and encodes the P50 subunit of NFкB (Inoue et al., 1992; Héron et al., 1995; Pereira and Oakley, 2008) (Fig. 1). The p50 homodimer represses transcription of pro-inflammatory cytokines, such as TNF- $\alpha$ and IL-12, and stimulates transcription of the anti-inflammatory cytokine IL10 (Pereira and Oakley, 2008). A common insertion (ins)/ deletion (del) ( -94 ins/del ATTG rs28362491) polymorphism of NFKB1 gene promoter exerts functional effects on the transcription of the NFKB1 (Karban et al., 2004). Since the 4bp ins/del polymorphism produces a relatively large sequence change and its location is proximal to binding sites that are important to promoter regulation, the ATTG deletion (D) allele displays significantly reduced promoter activity and it is also involved in lower levels of p50 protein. Thus, this polymorphism seems to be relative to several inflammatory diseases such as ulcerative colitis, colorectal cancer, and rheumatoid arthritis (Karban et al., 2004; Song et al., 2011; López-Mejías et al., 2012). Recently, a close correlation between an NFKB1 gene promoter variant and a higher incidence of CAD was reported in a prospective study involving three independent healthy Caucasian populations (Vogel et al., 2011), indicating a potential linkage of NFKB1 gene polymorphism and the risk of $\mathrm{CAD}$ in the general population.

The aim of the present study was to assess the association between the polymorphism (rs28362491) of the NFKB1 gene and CAD using a case-control study in the Han and Uygur populations in China.

\section{Materials and Methods}

\section{Ethics approval of the study protocol}

Written informed consent was obtained from each participant after a full explanation of this study. All participants explicitly provided permission for DNA analyses and collection of clinical data. This study was conducted according to the standards of the Declaration of Helsinki, and the study was approved by the Ethics Committee of the First Affiliated Hospital of Xinjiang Medical University.

\section{Study population}

From January 2006 to December 2012, 1070 patients were recruited from the First Affiliated Hospital of Xinjiang Medical University. This study population consisted of 633 Han patients (405 men and 228 women) and 437 Uygur patients (336 men and 101 women) with CAD. The diagnostic criteria of CAD were defined as the presence of at least one significant coronary artery stenosis of more than $50 \%$ luminal diameter on coronary angiography. CAD patients who had a congenital hypercoagulable status with proven diseaselimiting life expectancy, impaired renal function, malignancy, connective tissue disease, or chronic inflammatory disease were excluded from the study.

The control (non-CAD) participants of 616 Han (409 men and 207 women) and 356 Uygur Chinese (235 men and 121 women) were selected from the Cardiovascular Risk Survey (Xie et al., 2010; Pan et al., 2013b). This study consists of 14,618 subjects and is a multiple-ethnic, community-based, cross-sectional study designed to investigate the prevalence, incidence, and risk factors for cardiovascular diseases in the Han, Uygur, and Kazakh populations in Xinjiang (west China) from June 2007 to March 2010. These subjects did not have any of the following conditions related to CAD: a positive family history, stable and unstable angina, myocardial infarction, evidence of CAD by electrocardiography and angiography, abnormality of regional wall motion, or relevant

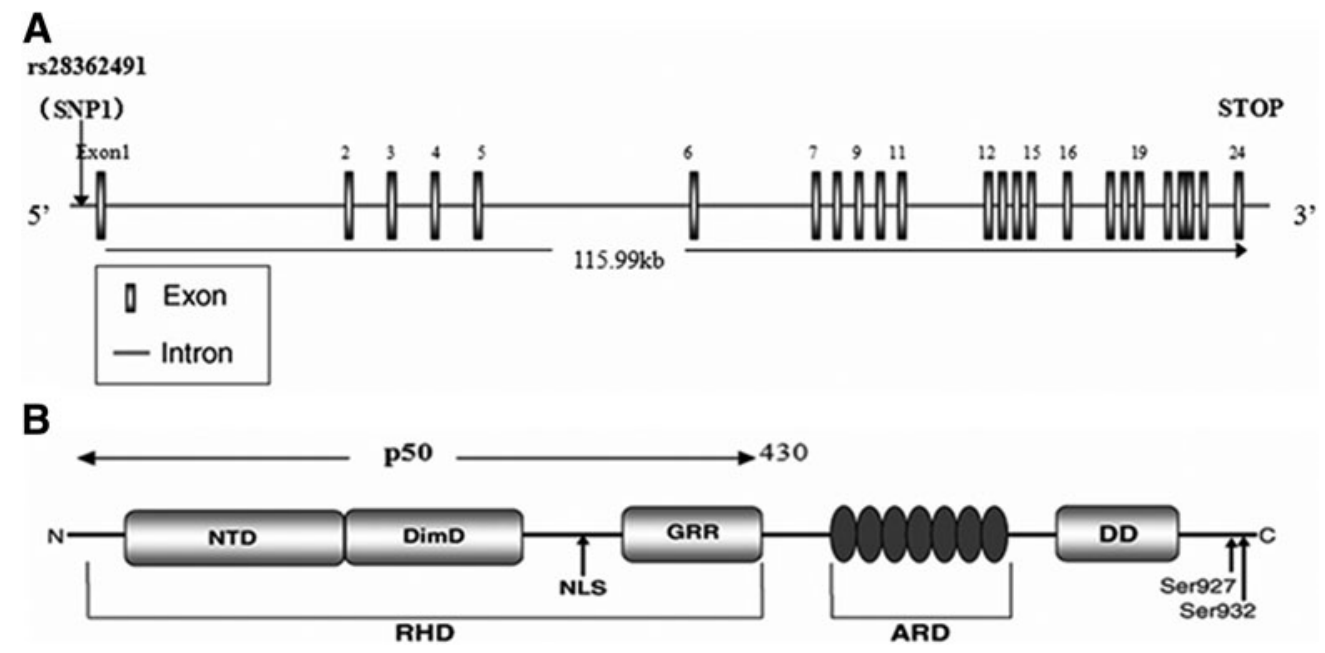

FIG. 1. NFKB1 gene and protein structures. (A) NFKB1 gene is $115.99 \mathrm{~kb}$ long and encodes a $3452 \mathrm{bp}$-long transcript containing 24 exons. (B) Protein structure of p50, p50 spans amino acids 1-430, which encompass the RHD; in this form, the NLS is exposed and promotes p50 nuclear translocation. ARD, ankyrin repeat domain; C, C-terminal; DD, death domain; GRR, glycine-rich region; NLS, nuclear localization sequence; NTD, N-terminal domain; RHD, Rel homology domain; N, N-terminal. 
valvular abnormalities in echocardiography (Guidelines Subcommittee, 1999). Both patients and control subjects were matched for age, gender, and ethnicity.

\section{Data collection}

Clinical data and information about the presence of traditional CAD risk factors, including diabetes mellitus (DM), essential hypertension (EH), total cholesterol (TC), smoking, and drinking, were obtained from all study participants by reviewing the patients' medical records. Diabetes was defined as fasting plasma glucose levels $\geq 7.0 \mathrm{mmol} / 1(126 \mathrm{mg} /$ $\mathrm{dL})$, glucose levels $\geq 11.1 \mathrm{mmol} / 1(200 \mathrm{mg} / \mathrm{dL})$ at $2 \mathrm{~h}$ after administration of $75 \mathrm{~g}$ of oral glucose load, a history of diabetes, or patients using anti-diabetic medication (World health organization study group, 1985). Hypertension was defined as having a systolic blood pressure above $140 \mathrm{mmHg}$ and/or diastolic blood pressure above $90 \mathrm{mmHg}$ at three consecutive measurements measured during 15 min intervals or the need for any antihypertensive agent (Guidelines subcommittee, 1999). Hypercholesterolemia was defined as TC levels $\geq 6.2 \mathrm{mmol} / 1$ ( $240 \mathrm{mg} / \mathrm{dL})$, triglyceride (TG) levels $\geq 2.26 \mathrm{mmol} / 1$, or current use of antihyperlipidemic medication. Participants were considered smokers when consuming more than five cigarettes per day or nonsmokers when they had never smoked or had stopped smoking at least 1 year before sample collection. Patients who had been drinking $100 \mathrm{~g}$ or more alcohol per day in the previous 6 months were considered alcohol users. The height and weight of each individual were recorded to calculate the body mass index (BMI) to determine the risk of obesity. BMI was calculated as weight divided by height squared $\left(\mathrm{kg} / \mathrm{m}^{2}\right)$.

\section{Blood collection and DNA extraction}

Blood samples were collected from all subjects using a standard venipuncture technique via ethylene diamine tetraacetic acid (EDTA)-containing tubes and centrifuged at 4000 $g$ for $5 \mathrm{~min}$ to separate the plasma for a range of biochemical assays. DNA was extracted from the peripheral vein blood leukocytes using a whole-blood genome extraction kit (Beijing Bioteke Corporation, Beijing, China. http://bioteke .technew.cn). The DNA sample was stored at $-80^{\circ} \mathrm{C}$ for genotyping.

\section{Biochemical measurements}

Plasma concentrations of TC, TG, high-density lipoprotein cholesterol (HDL-C), low-density lipoprotein cholesterol (LDL-C), glucose (Glu), blood urea nitrogen (BUN), creatinine $(\mathrm{Cr})$, and uric acid (UA) were measured using standard methods in the Central Laboratory of the First Affiliated Hospital of Xinjiang Medical University.

\section{Genotyping}

First, we titrated the DNA concentration at $50 \mathrm{ng} / \mu \mathrm{L}$. The - 94 ATTG ins/del (rs28362491) polymorphism of NFKB1 gene was detected by TaqMan single nucleotide polymorphism (SNP) genotyping assays in a 7900 HT Standard RealTime polymerase chain reaction (PCR) system, according to the manufacturer's recommendations (Applied Biosystems). Primers and probes used in the TaqMan SNP Genotyping
Assays (Applied Biosystems) were chosen based on information available at the ABI website (www3.appliedbiosystems.com/AB_Home/index.htm) (Pan et al., 2013a). PCR amplification was performed in a total reaction volume of $6 \mu \mathrm{L}$ that contains $3 \mu \mathrm{L}$ of TaqMan Universal Master Mix, $0.15 \mu \mathrm{L}$ probes, $1.85 \mu \mathrm{L} \mathrm{ddH}_{2} \mathrm{O}$, and $1 \mu \mathrm{L}$ DNA with the following amplification protocol: $95^{\circ} \mathrm{C}$ for $10 \mathrm{~min}, 40$ cycles of $95^{\circ} \mathrm{C}$ for $15 \mathrm{~s}$, and $60^{\circ} \mathrm{C}$ for $1 \mathrm{~min}$. All 96-well plates were read on the Sequence Detection Systems automation controller software v2.4 (ABI). Variant in the SNP (rs28362491) of the NFKB1 gene promoter was classified into three genotypes: DD, ATTG deleted homozygote; ID, ATTG inserted and deleted heterozygote and II, ATTG inserted homozygote.

\section{Statistical analysis}

Continuous statistics were presented as mean and standard deviation (mean $\pm \mathrm{SD}$ ), and differences in continuous variables between the CAD patients and control subjects were analyzed using an independent-sample $t$-test. The Hardy-Weinberg equilibrium was assessed by Chi-square analysis. Differences in enumeration data between CAD patients and control subjects were analyzed using the Chi-square test or Fisher's exact test. The differences in genotype and allele frequency between study groups were estimated by the Chi-square test. Logistic regression analyses with effect ratios (odds ratio [OR] and $95 \%$ confidence interval $[\mathrm{CI}]$ ) were used to assess the contribution of the major risk factors. All statistical analyses were performed using SPSS 17.0 for Windows (SPSS Institute). $p<0.05$ was considered statistically significant.

\section{Results}

The distribution of demographic characteristics of the study participants is shown in Tables 1 and 2. Overall, no significant difference was observed in age between CAD patients and control subjects. In the total Han population, the following variables were significantly different between CAD patients and control subjects: the plasma levels of Glu, TG, TC, HDL-C, and LDL-C and the prevalence of EH, DM, and drinking $(p<0.05$ in all). There was no significant difference in levels of BUN, Cr and UA, BMI, waist circumference (WC), and the prevalence of smoking between the two groups. In the male Han population, the following variables were significantly different between CAD patients and control subjects: the serum concentration of Glu, TG, TC, HDL-C, LDL-C, and $\mathrm{Cr}$; the prevalence of $\mathrm{EH}, \mathrm{DM}$ and drinking, WC and BMI $(p<0.05$ in all). There was no significant difference in levels of BUN and UA and the prevalence of smoking between the two groups. In the female Han population, the following variables were significantly different between CAD patients and control subjects: Glu, HDL-C, and the prevalence of EH and DM $(p<0.05$ in all). There was no significant difference in levels of BUN, Cr, UA, TG, TC, and LDL-C; the prevalence of smoking and drinking; or BMI and WC between the two groups.

In the Uygur population, the following variables were significantly different between CAD patients and control subjects: the plasma levels of Glu, TG, HDL-C, and UA; the prevalence of $\mathrm{EH}, \mathrm{DM}$, smoking and drinking, BMI, and $\mathrm{WC}$ $(p<0.05$ in all). There was no significant difference in levels of BUN, Cr, TC, and LDL-C between the two groups. In the male Uygur population, the following variables were 


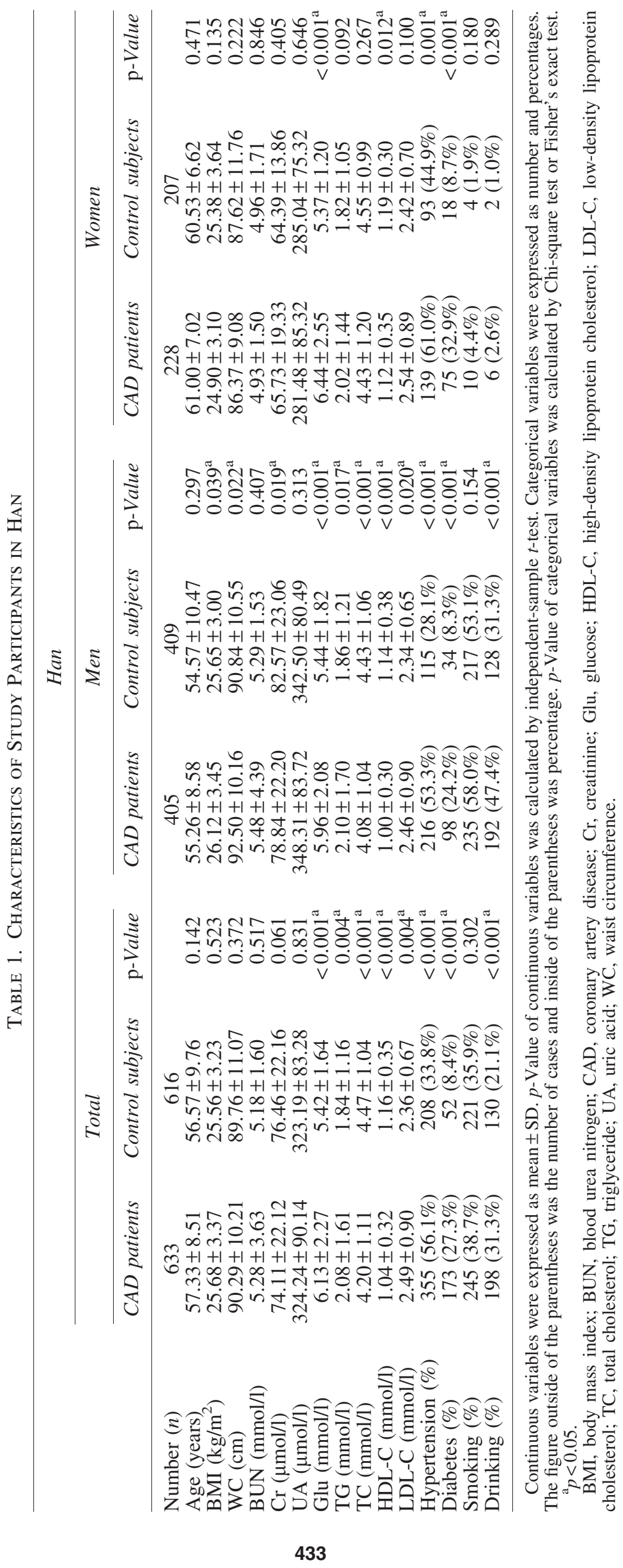




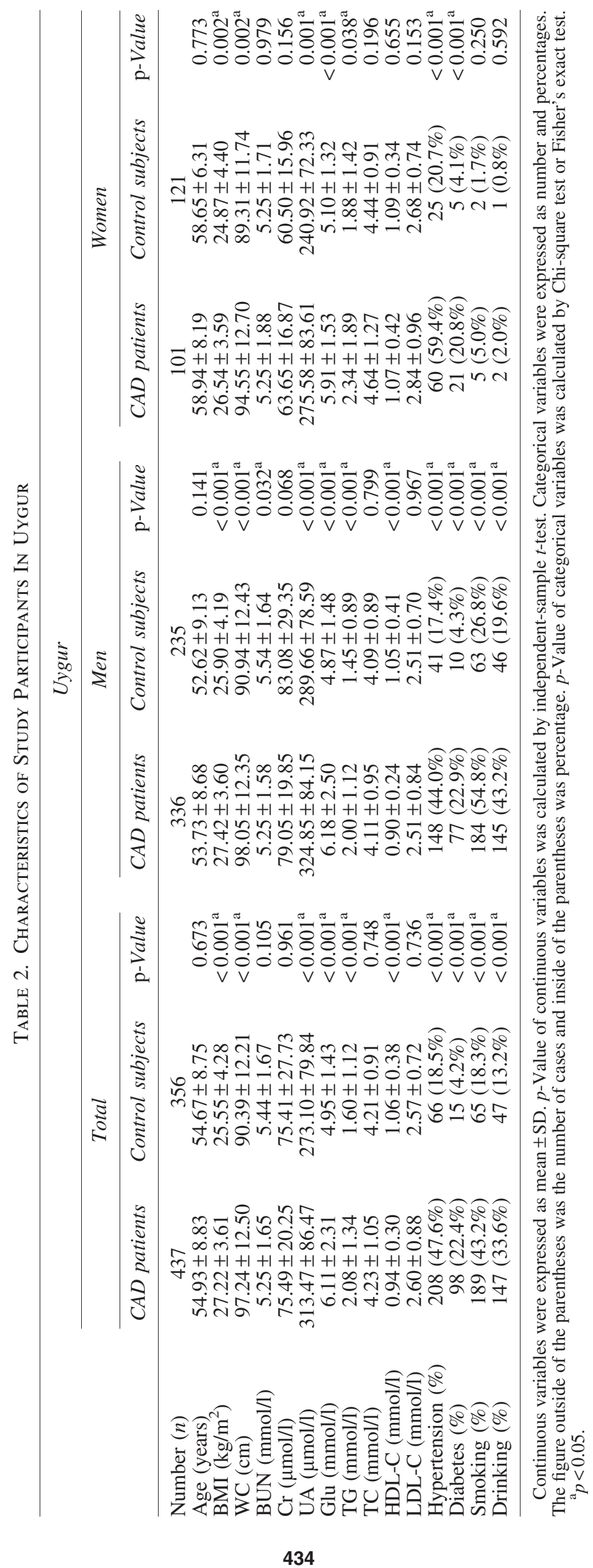



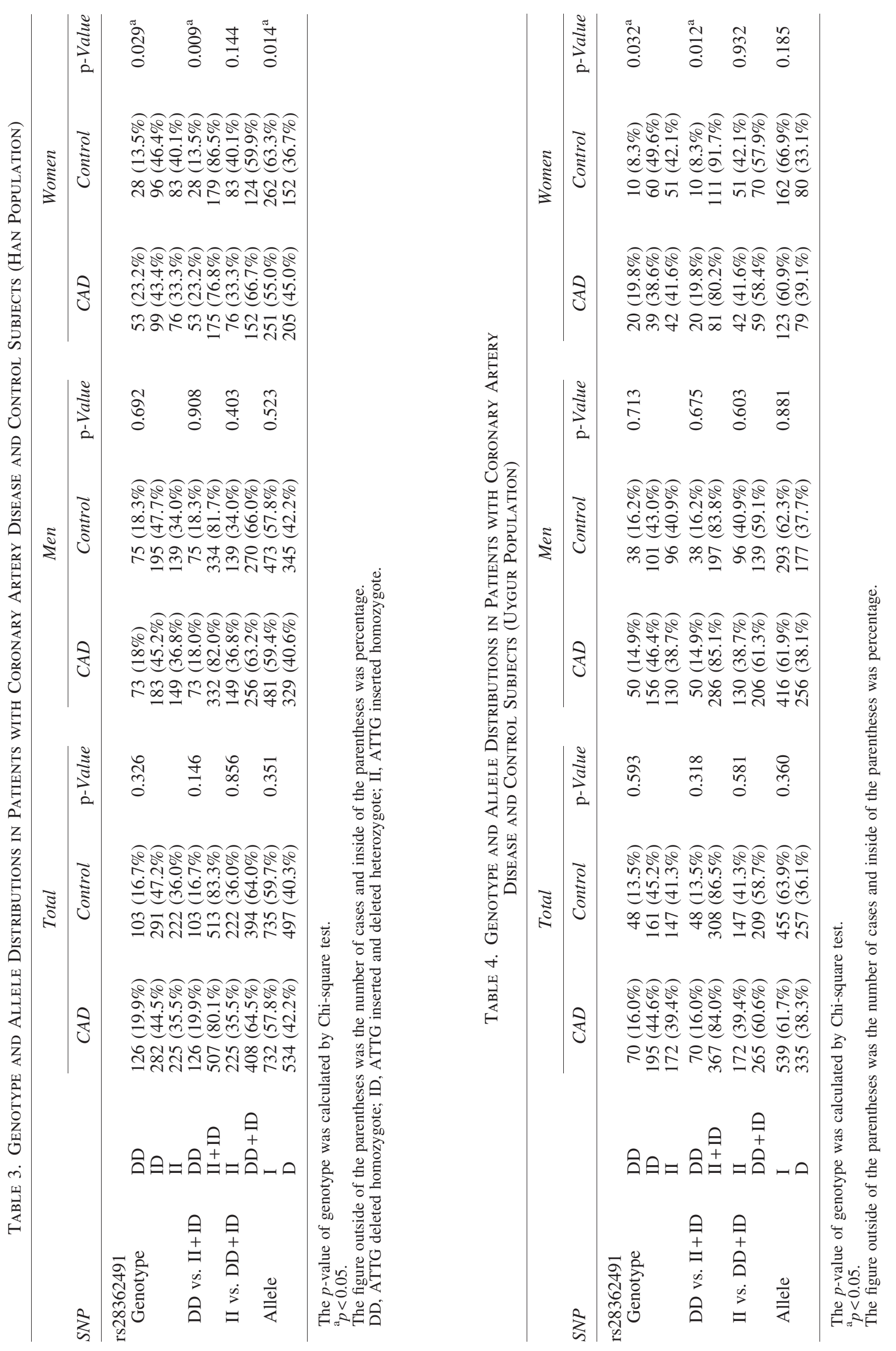
Table 5. Multiple Logistic Regression Analysis for Coronary Artery Disease Patients and Control Subjects in Han and Uygur Women

\begin{tabular}{|c|c|c|c|c|c|c|}
\hline \multirow[b]{2}{*}{ Risk factor } & \multicolumn{3}{|c|}{ Han women } & \multicolumn{3}{|c|}{ Uygur women } \\
\hline & $O R$ & $95 \% C I$ & p-Value & $O R$ & $95 \% C I$ & p-Value \\
\hline Genotype (DD vs. ID + II) & 1.805 & $1.062-3.067$ & $0.029^{\mathrm{a}}$ & 3.192 & $1.298-7.851$ & $0.011^{\mathrm{a}}$ \\
\hline Smoking & 1.847 & $0.508-6.717$ & 0.352 & 2.105 & $0.307-14.451$ & 0.449 \\
\hline Drinking & 2.528 & $0.462-13.827$ & 0.285 & 2.418 & $0.131-44.739$ & 0.553 \\
\hline $\mathrm{EH}$ & 1.551 & $1.031-2.335$ & $0.035^{\mathrm{a}}$ & 5.602 & $2.937-10.683$ & $<0.001^{\mathrm{a}}$ \\
\hline $\mathrm{DM}$ & 4.500 & $2.541-7.971$ & $<0.001^{\mathrm{a}}$ & 4.726 & $1.585-14.090$ & $0.005^{\mathrm{a}}$ \\
\hline $\mathrm{TC}$ & 1.095 & $0.910-1.318$ & 0.338 & 1.034 & $0.772-1.384$ & 0.823 \\
\hline
\end{tabular}

${ }^{\mathrm{a}} p<0.05$.

CI, confidence interval; DM, diabetes mellitus; EH, essential hypertension; OR, odds ratio.

significantly different between CAD patients and control subjects: the plasma levels of Glu, TG, HDL-C, BUN, and $\mathrm{UA}$; the prevalence of $\mathrm{EH}, \mathrm{DM}$, smoking and drinking, BMI, and $\mathrm{WC}(p<0.05$ in all $)$. There was no significant difference in levels of TC, LDL-C, and $\mathrm{Cr}$ between the two groups. In the female Uygur population, the following variables were significantly different between CAD patients and control subjects: the plasma levels of Glu, TG, and UA; the prevalence of essential EH and DM, BMI and WC ( $p<0.05$ in all). There was no significant difference in levels of BUN, Cr, TC, HDL-C and LDL-C, and the prevalence of drinking and smoking between the two groups.

Tables 3 and 4 show the frequency of genotypes and alleles for the SNP (rs28362491) of the NFKB1 gene. The genotype distribution for the SNP is in agreement with the predicted Hardy-Weinberg equilibrium values (data not shown). In the female Han and Uygur populations, the distribution of three SNP (rs28362491) genotypes (DD, ID, and II) in the NFKB1 gene was markedly different between CAD and control participants ( $p=0.029$ in Han and $p=0.032$ in Uygur). Further, the frequency of the DD homozygous carriers of the SNP (rs28362491) in the NFKB1 gene was significantly higher in CAD patients than in control subjects $(23.2 \%$ vs. $13.5 \%$ in female Han) and (19.8\% vs. $8.3 \%$ in female Uygur). In addition, the frequency of the model (DD vs. ID + II) (rs28362491) was also higher in CAD than in the control participants for both female ethnicities $(p=0.009$ in Han and $p=0.012$ in Uygur). In the female Han population, the D allele of SNP (rs28362491) was significantly higher in CAD patients than in control participants $(45.0 \%$ vs. $36.7 \%$; $p=0.014)$. However, in the male Han and Uygur populations, the distribution of the SNP genotypes and alleles showed no significant difference between the CAD patients and control subjects.

To further confirm the relationship between the frequency of the DD variant of the SNP (rs28362491) in the NFKB1 gene and CAD risk, multiple logistic regression analyses were performed by adjusting with smoking, drinking, EH, $\mathrm{DM}$, and TC, because these variables were the major confounding factors for CAD. For women, the significant difference in the frequency between DD and ID + II was retained after adjustment for these confounding factors in both the Han (OR: 1.805, 95\% CI: 1.062-3.067, $p=0.029$ ) and the Uygur (OR: 3.192, 95\% CI: 1.298-7.851, $p=0.011$ ) populations (Table 5), indicating that a greater frequency of DD carriers may have a higher risk to developing CAD.

\section{Discussion}

Over the past decade, enormous progress has been made in clarifying the regulatory mechanisms for CAD. CAD is a multifactorial disease, and its etiology and pathogenesis are likely polyfactorial due to the inheritance of several susceptibility genes, as well as multiple environmental determinants (Marenberg et al., 1994; Zee et al., 2006). To date, several genes and genetic variants are known to be related to CAD, but these explain only a minor portion of the etiology of CAD. In the present case-control study, we investigated the associations of the -94 ATTG ins/del polymorphism of the NFKB1 gene with the risk of CAD in the Han and Uygur populations in Xinjiang, the northwest part of China. Compared with the published data, our results indicate that the genotype distributions of the SNP (rs28362491) in the NFKB1 gene vary with ethnicity. Since the frequencies of the DD, ID and II genotypes of the NFKB1-94 ins/del ATTG in Han healthy subjects were $16.7 \%, 47.2 \%$, and $36.0 \%$, respectively, results were similar to a previous report of $17.7 \%, 45.9 \%$, and $36.4 \%$ in 412 Han Chinese (Zhang et al., 2013). However, in Uygur healthy subjects, frequencies of the DD, ID, and II genotypes were $13.5 \%$, $45.2 \%$, and $41.3 \%$, respectively, compared with $17 \%, 58 \%$, and $24 \%$ reported by Sun and Zhang (2007). It is likely that genetic differences among ethnicities may contribute to the discrepant results. Moreover, environmental influences such as diet and lifestyle may also contribute to the discrepancy (Huxley et al., 2009). However, this hypothesis warrants further investigation.

For women, the frequency of the DD genotype of the SNP (rs28362491) was significantly higher in CAD patients than in control subjects not only in the Han population but also in the Uygur population. However, a higher D-allele frequency of rs28362491 was only found in Han female CAD patients. Importantly, the significant difference was retained after adjustment for covariates such as smoking, drinking, EH, DM, and TC. These findings indicate an association between a higher frequency of the DD genotype of SNP (rs28362491) in the NFKB1 gene and increased risk of CAD in both female Han and Uygur populations. Only D-allele frequency of SNP (rs28362491) in the NFKB1 gene links with the risk of CAD in Han women. However, there is controversy among reports based on the concept that the NFKB1 polymorphism may contribute to the risk of CAD. Vogel et al. (2011) studied three independent Caucasian 
populations and found that carriers of the $\mathrm{D}$ allele of the functional NFKB1 ATTG ins/del promoter polymorphism had a higher risk of CAD than homozygous I carriers. However, another study reported by Boccardi et al. (2011) showed that D-allele carriers of - 94 ins/del ATTG NFKB1 gene variant tested from left ventricular biopsy had a lower susceptibility to myocardial infarction (MI) than those patients with MI. The reason for these distinct results is not clear but may be attributed to the difference in ethnicity, lifestyle, diet, and source of test samples.

Why does the high frequency of DD carriers of SNP (rs28362491) in the NFKB1 gene link to a high risk of CAD? What is the underlying mechanism? Multiple studies have demonstrated the important role of $\mathrm{NF}-\kappa \mathrm{B}$ in cardiac pathology (Hernández-Gutierrez et al., 2006; Kumar et al., 2013). CAD is recognized as a complex disease with a strong inflammatory component; particularly, inflammation has been found to play a critical role in all stages of atherogenesis (Weber and Noels, 2011). NF- $\mathrm{KB}$ is a ubiquitous dimeric transcription factor that regulates the expression of more than 160 target genes which are mainly involved in inflammatory response (Shapshak, 2012). The target gene specificity of NF$\kappa \mathrm{B}$ is determined by the subunit type. NF- $\kappa \mathrm{B}$ is involved in both pro-inflammatory and anti-inflammatory processes in atherogenesis (De Winther et al., 2005). The p50 encoded by NFKB1 gene has both pro- and anti-inflammatory functions. As a part of the p50/p65 NF- $\kappa \mathrm{B}$ transcription factor complex, it is pro-inflammatory, by stimulating the transcription of pro-inflammatory cytokines such as TNF- $\alpha$ and IL-1 $\beta$ (Perkins, 2007). In contrast, p50 has anti-inflammatory properties in the p50 homodimer (p50/p50), which represses transcription of pro-inflammatory cytokines such as TNF- $\alpha$ and IL-12 and stimulates transcription of the anti-inflammatory cytokine IL-10 (Cao et al., 2006). It has been shown that the deletion of the ATTG repeat in the promoter region of the NFKB1 gene results in lower promoter transcriptional activity and causes a decrease in p50 subunit levels (Karban et al., 2004). Therefore, it can affect both the availability of the pro-inflammatory $\mathrm{p} 50 / \mathrm{p} 65 \mathrm{NF}-\kappa \mathrm{B}$ heterodimer and antiinflammatory p50/p50 NFאB homodimer. However, for the DD genotype, since the low p50 levels will intuitively affect the concentration of p50/p50 more than the concentration of $\mathrm{p} 50 / \mathrm{p} 65$, the combined effect of relatively low levels of both p50/p50 and p50/p65 will be pro-inflammatory (Stegger et al., 2013). Consequently, the p50 ability to repress transcription of pro-inflammatory cytokines and its ability to stimulate transcription of the anti-inflammatory cytokines could be disturbed, thereby resulting in a higher pro-inflammatory status that may exaggerate the progression of coronary artery atherosclerosis.

In conclusion, our study demonstrated the association between - 94 ins/del ATTG polymorphism of the NFKB1 gene and the risk of CAD in female Han and Uygur populations in the northwest part of China. The DD genotype of SNP (rs28362491) in the NFKB1 gene could be considered a genetic marker for CAD risk among Han and Uygur women. The D allele may be considered a genetic marker for risk of CAD among women of the Han population. However, additional studies on a larger scale and in different populations with more detailed data on environment exposure are required to verify these findings.

\section{Source of Fundings}

This work was supported by the Program of Natural Science Fund of China (serial number: 81160042) and the Excellent Talents Plan of New Century (serial number: NCET11-1074).

\section{Author Disclosure Statement}

No competing financial interests exist.

\section{References}

Boccardi V, Rizzo MR, Marfella R (2011) - 94 ins/del ATTG NFKB1 gene variant is associated with lower susceptibility to myocardial infarction. Nutr Metab Cardiovasc Dis 21: 679-684.

Cao S, Zhang X, Edwards JP, et al. (2006) NF-kappa B1 (p50) homodimers differentially regulate pro- and anti-inflammatory cytokines in macrophages. J Biol Chem 281:2604126050.

Chen F, Castranova V, Shi X, et al. (1999) New insights into the role of nuclear factor-kappaB, a ubiquitous transcription factor in the initiation of diseases. Clin Chem 45:7-17.

De Winther MP, Kanters E, Kraal G, et al. (2005) Nuclear factor $\kappa \mathrm{B}$ signaling in atherogenesis. Arterioscler Thromb Vasc Biol 25:904-914.

Dhingra R, Shaw JA, Aviv Y, et al. (2010) Dichotomous actions of NF- $\kappa \mathrm{B}$ signaling pathways in Heart. J Cardiovasc Transl Res 3:344-354.

Guidelines Subcommittee (1999) World Health OrganizationInternational Society of Hypertension guidelines for the management of hypertension. J Hypertens 17:151-183.

Hajer GR, van Haeften TW, Visseren FL (2008) Adipose tissue dysfunction in obesity, diabetes, and vascular diseases. Eur Heart J 29:2959-2971.

Hernández-Gutierrez S, García-Peláez I, Zentella-Dehesa A,

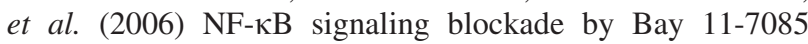
during early cardiac morphogenesis induces alterations of the outflow tract in chicken heart. Apoptosis 11:1101-1109.

Héron E, Deloukas P, van Loon AP (1995) The complete exonintron structure of the $156-\mathrm{kb}$ human gene NFKB1, which encodes the p105 and p50 proteins of transcription factors NF-kappa B and I kappa B-gamma: implications for NFkappa B-mediated signal transduction. Genomics 30:493505.

Huxley RR, Ansary-Moghaddam A, Clifton P, et al. (2009) The impact of dietary and lifestyle risk factors on risk of colorectal cancer: a quantitative overview of the epidemiological evidence. Int J Cancer 125:171-180.

Inoue J, Kerr LD, Kakizuka A, et al. (1992) I kappa B gamma, a $70 \mathrm{kd}$ protein identical to the C-terminal half of p110 NFkappa B: a new member of the I kappa B family. Cell 68: 1109-1120.

Kampoli AM, Tousoulis D, Antoniades C, et al. (2009) Biomarkers of premature atherosclerosis. Trends Mol Med 15: 323-332.

Karban AS, Okazaki T, Panhuysen CI, et al. (2004) Functional annotation of a novel NFKB1 promoter polymorphism that increases risk for ulcerative colitis. Hum Mol Genet 13:35-45.

Kumar R, Yong QC, Thomas CM (2013) Do multiple nuclear factor kappa B activation mechanisms explain its varied effects in the heart? Ochsner J 13:157-165.

Libby P (2012) History of discovery: inflammation in atherosclerosis. Arterioscler Thromb Vasc Biol 32:2045-2051. 
Libby P, Ridker PM, Maseri A (2002) Inflammation and atherosclerosis. Circulation 105:1135-1143.

López-Mejías R, García-Bermúdez M, González-Juanatey C, et al. (2012) NFKB1 -94ATTG ins/del polymorphism (rs28362491) is associated with cardiovascular disease in patients with rheumatoid arthritis. Atherosclerosis 224:426-429.

Marenberg ME, Risch N, Berkman LF, et al. (1994) Genetic susceptibility to death from coronary heart disease in a study of twins. N Engl J Med 330:1041-1046.

Pan S, Nakayama T, Sato N, et al. (2013a) A Haplotype of the GOSR2 gene is associated with myocardial infarction in Japanese men. Genet Test Mol Biomarkers 17:481-488.

Pan S, Yu Z, Ma Y, et al. (2013b) Appropriate body mass index and waistcircumference cutoffs for categorization of overweight and central adiposity among Uighur adults in Xinjiang. PLoS One 8:e80185.

Pereira SG, Oakley F (2008) Nuclear factor-kappaB1: regulation and function. Int J Biochem Cell Biol 40:1425-1430.

Perkins ND (2007) Integrating cell-signalling pathways with NF- $\kappa \mathrm{B}$ and IKK function. Nat Rev Mol Cell Biol 8:49-62.

Santos DG, Resende MF, Mill JG, et al. (2010) Nuclear Factor (NF) $\kappa \mathrm{B}$ polymorphism is associated with heart function in patients with heart failure. BMC Med Genet 11:89.

Shapshak P (2012) Bioinformation and molecule of the month: multiple interactive pathways for a single gene, NF- $\kappa$ B. Bioinformation 8:399-402.

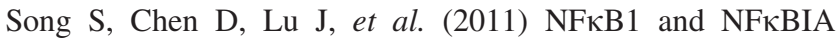
polymorphisms are associated with increased risk for sporadic colorectal cancer in a southern Chinese population. PLoS One 6:e21726.

Stegger JG, Schmidt EB, Berentzen TL, et al. (2013) Interaction between obesity and the NFKB1-94ins/del ATTG promoter polymorphism in relation to incident acute coronary syndrome: a follow up study in three independent cohorts. PLoS One 8:e63004.

Sun XF, Zhang H (2007) NFKB and NFKBI polymorphisms in relation to susceptibility of tumour and other diseases. Histol Histopathol 22:1387-1398.
Van der Heiden K, Cuhlmann S, Luong le A, et al. (2010) Role of nuclear factor kappaB in cardiovascular health and disease. Clin Sci (Lond) 118:593-605.

Vogel U, Jensen MK, Due KM, et al. (2011) The NFKB1 ATTG ins/del polymorphism and risk of coronary heart disease in three independent populations. Atherosclerosis 219:200-204.

Vogiatzi G, Tousoulis D, Stefanadis C (2009) The role of oxidative stress in atherosclerosis. Hellenic J Cardiol 50:402-409.

Weber C, Noels H (2011) Atherosclerosis: current pathogenesis and therapeutic options. Nat Med 17:1410-1422.

World Health Organization Study Group (1985) Diabetes mellitus. WHO Tech Rep Ser 727:1-104.

Xie X, Ma YT, Yang YN, et al. (2010) Polymorphisms in the SAA1/2 gene are associated with carotid intima media thickness in healthy Han Chinese subjects: the Cardiovascular Risk Survey. PLoS One 5:e13997.

Zee RY, Cheng S, Hegener HH, et al. (2006) Genetic variants of arachidonate 5-lipoxygenase-activating protein, and risk of incident myocardial infarction and ischemic stroke: a nested case-control approach. Stroke 37:2007-2011.

Zhang DY, Li LB, Zhu YJ, et al. (2013) The NFKB1 -94 ATTG insertion/deletion polymorphism (rs28362491) contributes to the susceptibility of congenital heart disease in a Chinese population. Gene 516:307-310.

Zhou B, Rao L, Peng Y, et al. (2009) Functional polymorphism of the NFKB1 gene promoter is related to the risk of dilated cardiomyopathy. BMC Med Genet 10:47.

Address correspondence to: Yi-Tong $M a, M D, P h D$ Department of Cardiology First Affiliated Hospital of Xinjiang Medical University Li Yu Shan South Road 137 Urumqi 830054 China

E-mail:myt_xj@sina.com 